\section{Fatal Invasive Aspergillosis and Coronavirus Disease in an Immunocompetent Patient}

\author{
Marion Blaize, Julien Mayaux, Cécile Nabet, \\ Alexandre Lampros, Anne-Geneviève Marcelin, \\ Marc Thellier, Renaud Piarroux, Alexandre Demoule, \\ Arnaud Fekkar
}

Author affiliations: Assistance Publique-Hôpitaux de Paris, Groupe Hospitalier La Pitié-Salpêtrière, Paris, France (M. Blaize, J. Mayaux, C. Nabet, A. Lampros, A.-G. Marcelin, M. Thellier, R. Piarroux, A. Demoule, A. Fekkar); Sorbonne Université, Paris (C. Nabet, A.G. Marcelin, M. Thellier, R. Piarroux, A. Fekkar)

\section{DOI: https://doi.org/10.3201/eid2607.201603}

Invasive pulmonary aspergillosis is a complication in critically ill patients with acute respiratory distress syndrome, especially those with severe influenza pneumonia. We report a fatal case of invasive pulmonary aspergillosis in an immunocompetent patient in France who had severe coronavirus disease-associated pneumonia.

Datic atients infected with severe acute respiratory syndrome coronavirus 2 (SARS-CoV-2) experience major lung damage due to viral replication and the ensuing cytokine storm and complex inflammatory processes (1). The severe damage to lung tissue can lead to secondary infections within a median of 17 days after onset of coronavirus disease (COVID-19) (2). Most immunocompetent patients who develop severe forms of COVID-19 have $\geq 1$ underlying condition, such as chronic obstructive pulmonary disease, hypertension, diabetes, or chronic kidney disease (3), but none of these predisposing factors generally are associated with an increased risk for developing fungal infections.

Invasive aspergillosis is a well-described complication of severe influenza pneumonia $(4,5)$, but many intensivists seem to overlook this superinfection (6). A recent study reported a 19\% incidence among 432 patients admitted to an intensive care unit (ICU) for influenza-related acute respiratory failure (4). Moreover, in a small autopsy series of patients who died in 2003 from SARS, 10\% (2/20) had an invasive infection suggestive of aspergillosis (7). As the medical community confronts the ongoing COVID-19 pandemic, determining whether patients infected with SARS$\mathrm{CoV}-2$ develop fungal complications, especially invasive aspergillosis, is crucial.

We report the case of a 74-year-old immunocompetent man with severe COVID-19-associated pneumonia who rapidly developed invasive pulmonary aspergillosis. The patient had several underlying chronic diseases but no pulmonary disease. Among his existing conditions were asymptomatic and untreated myelodysplastic syndrome, diagnosed on the basis of hypereosinophilia, with CD8+ T-cell lymphocytosis, a normal karyotype and low-risk International Prognostic Scoring System score; Hashimoto's thyroiditis; hypertension; and benign prostatic hypertrophy.

On the morning of March 23, day 1, the patient fell out of bed, was unable to get up on his own, and called emergency services. The patient described a 1-week history of fever and cough followed by onset of dyspnea that day. Clinical examination showed signs of acute respiratory failure, tachypnea, and hypoxemia. The patient was intubated, transported to the hospital, and transferred to the ICU, where he required mechanical ventilation and vasopressor support. The same day, we took a protected distal aspiration before intravenous administration of cefotaxime. SARS-CoV-2 viral RNA was detected in this sample by using 2 distinct reverse transcription PCRs. The sample revealed $10^{4} / \mathrm{mL}$ of Haemophilus influenzae, which was not found in a second protected distal aspiration performed on day 2 .

The patient's condition worsened, and he had a $\mathrm{PaO}_{2} / \mathrm{FIO}_{2}$ ratio $<140 \mathrm{~mm} \mathrm{Hg}$ with $\mathrm{FIO}_{2}$ of $100 \%$. We performed a tracheal aspirate on day 4 and sent samples to the mycology laboratory. PCR for Aspergillus fumigatus was positive (430 copies/mL; $2.6 \mathrm{log}$ ), but galactomannan in the aspirate assay was negative, and culture on Sabouraud agar remained sterile. Results of testing of a second tracheal aspiration performed on day 9 yielded branched septate hyphae suggestive of Aspergillus sp. under microscopic examination after silver staining (Figure). A. fumigatus PCR was once again positive with a higher number of copies $(3,600$ copies $/ \mathrm{mL} ; 3.55 \mathrm{log})$; the galactomannan assay was not available. The patient died that day of severe respiratory failure. Culture on Sabouraud agar later grew a mold identified as A. fumigatus by mass spectrometry. Of note, SARS-CoV-2 RNA still was detectable in the tracheal aspirations. Results of serum analyses, including galactomannan index determination, $\beta$-D-glucan assay, and $A$. fumigatus PCR, were negative in serum samples collected on day 4 and day 8 .

This case fulfills the criteria of putative invasive aspergillosis according to the classification defined by Blot et al. for the diagnosis of invasive pulmonary aspergillosis in critically ill patients (8). Because the patient's illness took a rapid and fatal course, we did not have time to initiate appropriate antifungal treatment. As previously reported, respiratory samples 


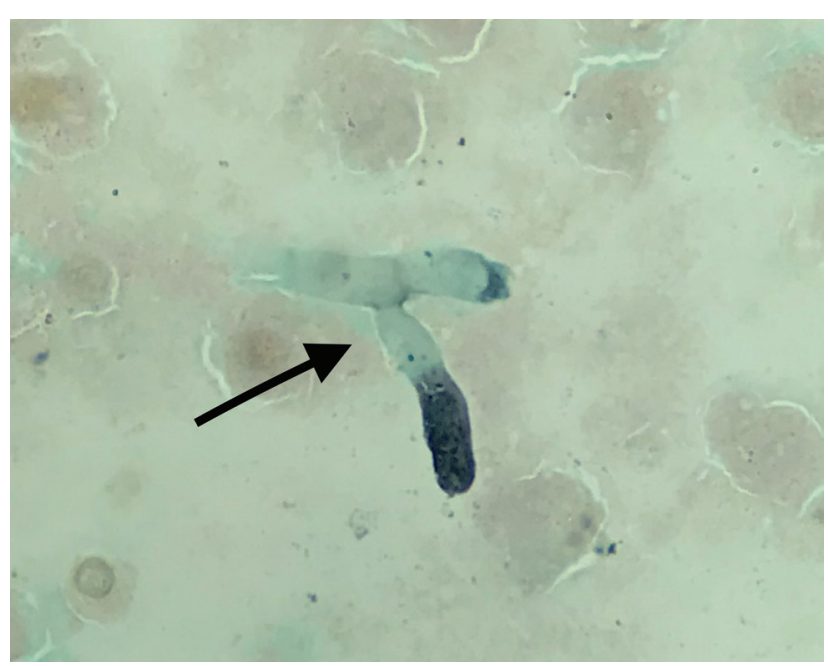

Figure. Microscopic image of silver-stained tracheal aspirate from an immunocompetent patient critically ill with coronavirus disease, France. Filaments suggestive of Aspergillus sp. are shown (black arrow). Original magnification $\times 1,000$.

led to a diagnosis of aspergillosis, but blood samples lacked sensitivity (4). Moreover, Aspergillus PCR contributed to the diagnosis, but the galactomannan was negative, consistent with what we previously reported about the lower sensitivity of the galactomannan compared with PCR and culture in respiratory samples (9).

A patient with a fatal SARS-CoV-2 infection recently was reported to have $A$. flavus on tracheal aspirates culture, but other evidence was missing, and diagnosis of aspergillosis was not substantiated (10). Additional assessments are urgently needed to thoroughly investigate cases and promptly establish and define the cumulative incidence of invasive aspergillosis in ICU patients with severe COVID-19.

In conclusion, we report a case of invasive pulmonary aspergillosis in an immunocompetent patient during severe COVID-19-associated pneumonia. As the COVID-19 outbreak continues to spread worldwide, other reports are needed to assess the occurrence and frequency of fungal complications during severe SARS-CoV-2 infections.

\section{About the Author}

Dr. Blaize is a microbiologist at Centre Hospitalier

Universitaire La Pitié-Salpêtrière, Paris, France. Her primary research interest is medical diagnosis of fungal diseases.

\section{References}

1. Mehta P, McAuley DF, Brown M, Sanchez E, Tattersall RS, Manson JJ; HLH Across Speciality Collaboration, UK. COVID-19: consider cytokine storm syndromes and immunosuppression. Lancet. 2020;395:1033-4. https://doi.org/10.1016/S0140-6736(20)30628-0

2. Zhou F, Yu T, Du R, Fan G, Liu Y, Liu Z, et al. Clinical course and risk factors for mortality of adult inpatients with COVID-19 in Wuhan, China: a retrospective cohort study. Lancet. 2020;395:1054-62. https:/ / doi.org/10.1016/ S0140-6736(20)30566-3

3. Onder G, Rezza G, Brusaferro S. Case-fatality rate and characteristics of patients dying in relation to COVID-19 in Italy. JAMA. 2020 Mar 23 [Epub ahead of print]. https://doi.org/10.1001/jama.2020.4683

4. Schauwvlieghe AFAD, Rijnders BJA, Philips N, Verwijs R, Vanderbeke L, Van Tienen C, et al.; Dutch-Belgian Mycosis study group. Invasive aspergillosis in patients admitted to the intensive care unit with severe influenza: a retrospective cohort study. Lancet Respir Med. 2018;6:782-92. https://doi.org/10.1016/S2213-2600(18)30274-1

5. Schwartz IS, Friedman DZP, Zapernick L, Dingle TC, Lee N, Sligl W, et al. High rates of influenza-associated invasive pulmonary aspergillosis may not be universal: a retrospective cohort study from Alberta, Canada. Clin Infect Dis. 2020 Jan 6 [Epub ahead of print]. https://doi.org/ 10.1093/cid/ciaa007

6. Toda M, Beekmann SE, Polgreen PM, Chiller TM, Jackson BR, Beer KD. Knowledge of infectious disease specialists regarding aspergillosis complicating influenza, United States. Emerg Infect Dis. 2020;26:809-11. https://doi.org/10.3201/eid2604.190953

7. Hwang DM, Chamberlain DW, Poutanen SM, Low DE, Asa SL, Butany J. Pulmonary pathology of severe acute respiratory syndrome in Toronto. Mod Pathol. 2005;18:1-10. https:/ / doi.org/10.1038/modpathol.3800247

8. Blot SI, Taccone FS, Van den Abeele AM, Bulpa P, Meersseman W, Brusselaers N, et al.; AspICU Study Investigators. A clinical algorithm to diagnose invasive pulmonary aspergillosis in critically ill patients. Am J Respir Crit Care Med. 2012;186:56-64. https:/ / doi.org/10.1164/ rccm.201111-1978OC

9. Imbert S, Meyer I, Palous M, Brossas JY, Uzunov M, Touafek F, et al. Aspergillus PCR in bronchoalveolar lavage fluid for the diagnosis and prognosis of aspergillosis in patients with hematological and non-hematological conditions. Front Microbiol. 2018;9:1877. https:/ / doi.org/ 10.3389/fmicb.2018.01877

10. Lescure FX, Bouadma L, Nguyen D, Parisey M, Wicky PH, Behillil S, et al. Clinical and virological data of the first cases of COVID-19 in Europe: a case series. Lancet Infect Dis. 2020 Mar 27 [Epub ahead of print]. https://doi.org/10.1016/ S1473-3099(20)30200-0

Address for correspondence: Arnaud Fekkar, Service de Parasitologie-Mycologie, Pavillon Laveran, Hôpital de La PitiéSalpêtrière, Boulevard de l'Hôpital, 75013 Paris, France; email: arnaud.fekkar@aphp.fr 